\title{
Increased complements and high-sensitivity C-reactive protein predict heart failure in acute myocardial infarction
}

\author{
DANNI LIU ${ }^{1,2}, \mathrm{XIN} \mathrm{QI}^{2}$, QI LI ${ }^{2}$, WENJUN JIA ${ }^{2}$, LIPING WEI $^{2}$, \\ ANAN HUANG ${ }^{1,2}$, KEQIANG LIU ${ }^{2}$ and ZONGJIN LI ${ }^{1}$ \\ ${ }^{1}$ Department of Pathophysiology, School of Medicine, Nankai University, Tianjin 300071; \\ ${ }^{2}$ Department of Cardiology, Tianjin Union Medical Center, Tianjin 300121, P.R. China
}

Received August 16, 2016; Accepted September 28, 2016

DOI: $10.3892 /$ br.2016.793

\begin{abstract}
The aim of the present study was to investigate whether the serum levels of complements and high-sensitivity C-reactive protein (hs-CRP) in patients with acute myocardial infarction (AMI) are associated with the severity of myocardial injury. Consecutive patients $(\mathrm{n}=110)$ with AMI and 33 healthy individuals, who served as control subjects, were enrolled from May 2013 to February 2015. These patients were divided into two groups, those with ST segment elevation MI (STEMI) and those with non-ST segment elevation MI (NSTEMI). The patients with STEMI exhibited progression to diastolic dysfunction and heart failure. Furthermore, the results revealed that the level of serum complement and hs-CRP in patients with AMI increased rapidly when compared with the subjects from the control group, particularly in the STEMI patients, at different time-points. A statistically significant elevation of the complement and hs-CRP levels was observed at day 3 after AMI in the STEMI group. The activation of complement and hs-CRP following AMI may serve as a specific marker to successfully predict left ventricular dysfunction. Thus, biomarker-based approaches may be adopted to identify the severity of AMI with distinct pathophysiologic responses in order to rationally implement clinical therapeutic strategies.
\end{abstract}

\section{Introduction}

Acute myocardial infarction (AMI) is associated with considerable morbidity and mortality worldwide $(1,2)$. The majority of myocardial infarcts result from coronary atherosclerosis, complicated by acute rupture of an atherosclerotic plaque and

Correspondence to: Professor Xin Qi, Department of Cardiology, Tianjin Union Medical Center, 190 Jieyuan Road, Tianjin 300121, P.R. China

E-mail: nankaiqixin@163.com

Key words: complement protein, acute myocardial infarction, high-sensitivity C-reactive protein, electrocardiography, ST segment elevation, heart failure subsequent formation of coronary thrombus. Coronary artery occlusion causes ischemia of the anatomic region supplied by the vessel, ultimately leading to death of a large quantity of cardiomyocytes. Generally, the heart possesses little regenerative capacity; therefore, repair of the infarcted myocardium is dependent on the sequence of cellular events that lead to the formation of a collagen-based scar (3-5). The necrotic cardiomyocytes elicit an intense inflammatory cascade that serves to clear the infarct site from dead cells and matrix debris, and eventually contributes to replacement of damaged tissue with collagen-based scar tissue (6).

Cardiac injury generates endogenous signals that activate the innate and adaptive immune system; these molecules belong to the $\alpha 2$-macroglobulin family of mediators that warn the body of injury and are termed complement factors $\mathrm{C} 3, \mathrm{C} 4$, C5b9 (7). The complement system mediates inflammation by generating anaphylatoxins (to trigger chemotaxis and cell activation) and promoting phagocytosis, degranulation and cell lysis (8). Prolonging inflammatory signaling in the infarcted heart may have numerous consequences, including loss of cardiomyocytes, suppression of systolic function, enhanced matrix-degrading processes leading to chamber dilation, increased tissue breakdown causing loss of ventricular wall integrity and cardiac rupture, as well as extended fibrotic changes beyond the initial infarct. Based on this mechanism, it has been verified that the primitive innate immune system is activated in coronary heart disease (CHD) by the acute phase response components, of which high-sensitivity C-reactive protein (hs-CRP) increases the inflammatory response with high specificity. Human hs-CRP binds to damaged cells and activates the complement system by an alternate signaling pathway (9). Experimental models have indicated that complement component $\mathrm{C} 3$ presents in atherosclerotic plaques, and participates in activation of the inflammatory system and promotes the process of phagocytosis by macrophages (10). Various complements are directly or indirectly involved in myocardial cell lysis or damage via formation of the membrane attack complex in the terminal pathway (11).

Recent studies revealed that the systemic inflammatory response, including the cascade of inflammatory and cytokine complement activation, is key in the development of myocardial infarction (3). hs-CRP is a marker of inflammation that predicts incident myocardial infarction, recurrent events 
and mortality in patients with AMI. However, the association between serum complement C3, C4, C5b9 and hs-CRP changes in patients with AMI and the severity of myocardial injury remains unclear. The present study aims to investigate the changes in levels of serum complement $\mathrm{C} 3, \mathrm{C} 4, \mathrm{C} 5 \mathrm{~b} 9$ and hs-CRP, and probe the potential association between the differentially expressed complement proteins and the severity of tissue damage following AMI.

\section{Materials and methods}

Study population. The current study was approved by the Hospital Review Board, Tianjin Union Medical Center of Nankai University Affiliated Hospital (Tianjin, China). A total of 110 consecutive patients $[73$ males and 37 females; age 62 years (range, 50-74 years)] with a diagnosis of AMI from May 2013 to February 2015 whose age and gender matched, and who had no personal or family history of premature cardiovascular disease, were selected. In addition, 33 healthy individuals were enrolled to serve as the control group. AMI was confirmed according to current guidelines (12). Based on their electrocardiograms (ECGs), AMI patients were categorized into two groups: ST segment elevation MI (STEMI; $\mathrm{n}=51$ ) and non-ST segment elevation MI (NSTEMI; n=59) (13). The exclusion criteria included the following: Patients with previous myocardial infarction or heart failure, cardiomyopathy, pericarditis, cerebral vascular disease, pulmonary embolism, hepatic insufficiency, renal insufficiency, hyperthyroidism, anemia, hematologic disease, autoimmune disease or acute inflammation. Written informed consent was obtained from all participants.

Clinical and laboratory data collection. At the time of enrollment, a detailed clinical history was recorded, which included age, gender, body mass index (BMI), blood pressure, heart rate and coronary artery disease history. Blood samples for hematological parameters were obtained from all patients. Creatine kinase-MB (CK-MB) (14) and Cardiac troponin T were determined by an electrochemiluminescence immunoassay on the Elecsys 2010 system (Roche Diagnostics GmbH, Mannheim, Germany) (15). Levels of cholesterol, high-density lipoprotein (HDL) cholesterol and low-density lipoprotein (LDL) cholesterol, and triglycerides were measured with the use of standard hospital assays through enzymatic methods (Roche Diagnostics $\mathrm{GmbH}$ ) with freshly drawn lithium-heparin plasma samples. Blood samples were obtained from all participants at admission, and at days 1, 3 and 7. Serum levels of C3, $\mathrm{C} 4$ and hs-CRP were measured quantitatively by velocity scattering turbidimetry using a Siemens BN II automatic protein analyzer (Siemens AG, Munich, Germany) as previously described (16). In addition, enzyme linked immunosorbent assays (Kamiya Biomedial Co., Seattle, WA, USA) were performed to quantify the serum level of C5b9 (8).

Echocardiography. Echocardiographic examination was performed as previously described (17). M-mode, two-dimensional, and Doppler echocardiography analysis (Vevo 770; Visual Sonics Biotechnology Co., Toronto, ON, Canada) provided the left ventricular (LV) end-diastolic diameter (LVEDD) and LV end-systolic diameter (LVESD). The LV systolic function was measured as ejection fraction (LVEF) and fractional shortening (LVFS) according to the modified Simpson method (6).

Statistical analysis. Data analyses were performed using Graph Pad Prism 5.0 software (GraphPad Software Inc., La Jolla, CA, USA). For comparisons between the two groups, analysis of variance and one-way Student's t-test were used. Continuous variables were expressed as the mean \pm standard deviation and $\mathrm{P}<0.05$ was considered to indicate a statistically significant difference.

\section{Results}

Patient characteristics. Statistical analysis of clinical information from all participants (Table I) revealed significant differences in CK-MB, troponin $\mathrm{T}$ and LVEF between the AMI patients and control subjects $(\mathrm{P}<0.05)$. No significant differences were identified in age $(\mathrm{P}=0.054)$, gender $(\mathrm{P}=1.000)$, BMI $(\mathrm{P}=0.969)$, heart rate $(\mathrm{P}=0.191)$, systolic blood pressure $(\mathrm{P}=0.694)$, diastolic blood pressure $(\mathrm{P}=0.663)$, cholesterol $(\mathrm{P}=0.326)$, triglyceride $(\mathrm{P}=0.268)$, or HDL cholesterol $(\mathrm{P}=0.66)$ and LDL cholesterol $(\mathrm{P}=0.056)$ between the two groups.

Changes in serum levels of $C 3, C 4, C 5 b 9$ and $h s-C R P$. It has been proposed that complement proteins are crucial in the development of AMI. The aim of the current study was to investigate the association between serum complement and hs-CRP changes, and the severity of myocardial injury, thus the differential expression of complement proteins in patients with AMI was evaluated. This approach may be used to detect changes in cardiac damage subsequent to interventions in patients with myocardial injury. In the present study, the results revealed that the patients with AMI tended to exhibit markedly higher levels of $\mathrm{C} 3, \mathrm{C} 4, \mathrm{C} 5 \mathrm{~b} 9$ and hs-CRP when compared with the subjects from the control group at days 1, 3 and 7. On day 3, subsequent to infarction, a significant enhancement in C3, C4, C5b9 and hs-CRP serum levels was observed in patients with AMI (Fig. 1; $\mathrm{P}<0.05$ ). These results indicate that hs-CRP and activation of complement may be indicators of myocardiocyte damage.

Increased serum levels of $C 3, C 4, C 5 b 9$ and $h s-C R P$ in the STEMI subgroup. To date, a small number of studies have demonstrated the association between serum complement, hs-CRP and heart failure (8). In the present study, it was confirmed that there was a more rapid increase in serum levels of C3, C4, C5b9 and hs-CRP in patients with STEMI when compared with those with NSTEMI or the control subjects at different time points (Fig. 2). Activation of the complement system is an important aspect of the inflammatory process that regulates injury, repair and remodeling of the infarcted heart. Notably, the levels of serum C3, C4, C5b9 and hs-CRP in the STEMI/NSTEMI groups peaked at day 3. Furthermore, the levels of complement C3, C4, C5b9 and hs-CRP in the STEMI group were higher than those in the NSTEMI group. These data indicate that activation of complement proteins and hs-CRP in patients increases the risk of complication and severity of AMI. 
Table I. Baseline characteristics of the AMI and control groups.

\begin{tabular}{lccr}
\hline & \multicolumn{2}{c}{ Group } & \\
\cline { 2 - 4 } Variable & Control $(\mathrm{n}=33)$ & AMI $(\mathrm{n}=110)$ & P-value \\
\hline Age (years) & $57.64 \pm 10.48$ & $62.06 \pm 11.59$ & 0.054 \\
Gender (male/female) & $22 / 11$ & $73 / 37$ & 1.000 \\
BMI $\left(\mathrm{kg} / \mathrm{m}^{2}\right)$ & $24.42 \pm 3.03$ & $24.45 \pm 1.98$ & 0.969 \\
Systolic blood pressure (mmHg) & $131.30 \pm 24.16$ & $133.85 \pm 25.97$ & 0.694 \\
Diastolic blood pressure (mmHg) & $76.30 \pm 8.95$ & $75.38 \pm 14.26$ & 0.663 \\
Heart rate (bpm) & $72.79 \pm 12.38$ & $75.60 \pm 13.93$ & 0.191 \\
Cholesterol (mmol/l) & $4.19 \pm 0.45$ & $4.79 \pm 0.91$ & 0.326 \\
Triglyceride (mmol/l) & $0.97 \pm 0.31$ & $1.53 \pm 0.83$ & 0.268 \\
HDL cholesterol (mmol/l) & $1.31 \pm 0.30$ & $1.06 \pm 0.37$ & 0.66 \\
LDL cholesterol (mmol/l) & $2.65 \pm 0.34$ & $2.79 \pm 0.76$ & 0.056 \\
Troponin T (ng/ml) & $0.012 \pm 0.001$ & $9.15 \pm 1.24$ & 0.002 \\
Creatine kinase-MB (U/l) & $10.67 \pm 2.86$ & $200.48 \pm 64.0$ & 0.001 \\
LVEF $(\%)$ & $66.87 \pm 5.53$ & $49.00 \pm 6.29$ & 0.002 \\
\hline
\end{tabular}

Variables are expressed as the mean \pm standard deviation in each group and $\mathrm{P}<0.05$ was considered to indicate a statistically significant difference. AMI, acute myocardial infarction; BMI, body mass index; HDL, high-density lipoprotein; LDL, low-density lipoprotein; LVEF, left ventricular ejection fraction.
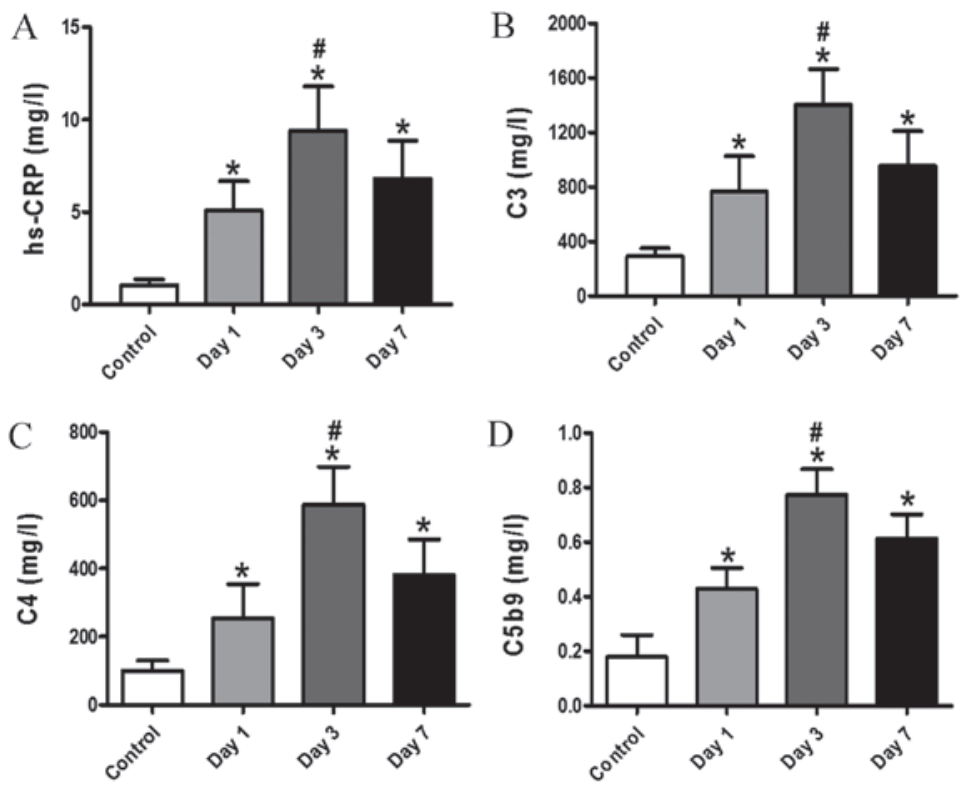

Figure 1. Changes in serum levels of complement and hs-CRP in AMI patients. (A) Quantitative analysis data reveal that serum hs-CRP levels of patients with AMI at day 3 was significantly higher than those on days 1 and 7 and in the control group. (B) Quantification of serum C3 levels. Patients with AMI on day 3 demonstrated significantly higher serum C3 levels on days 1 and 7, and compared with the control group. (C) Serum C4 levels of patients with AMI were measured at different time-points. (D) ELISA detection of C5b9 levels of patients with AMI in the serum samples on days 1, 3 and 7. Data are expressed as the mean \pm standard deviation. ${ }^{*} \mathrm{P}<0.05$ vs. control, ${ }^{*} \mathrm{P}<0.05$ vs. day 1 and day 7 . hs-CRP, high-sensitivity $\mathrm{C}$-reactive protein; AMI, acute myocardial infarction.

Heart function analysis. LV function was evaluated using echocardiograms on day 7 after infarction. Compared with the control groups, the patients with STEMI displayed more marked LV enlargement and impaired heart function than those with NSTEMI (Fig. 3A). Normal function in the myocardium is dependent on optimal preservation of structure, as minimal changes in cardiac morphology may exacerbate dysfunction. Subsequent to an infarction, the human heart undergoes a series of structural changes, which are governed by cytokines, complements and cellular mechanisms in a pathological metamorphosis. In the present study, patients with STEMI and NSTEMI demonstrated markedly increased LVESD and LVEDD of the heart when compared with the control group (Fig. 3B and C). In addition, patients with STEMI exhibited significantly exacerbated progression of LV dysfunction compared with the control group subjects, with decreased 

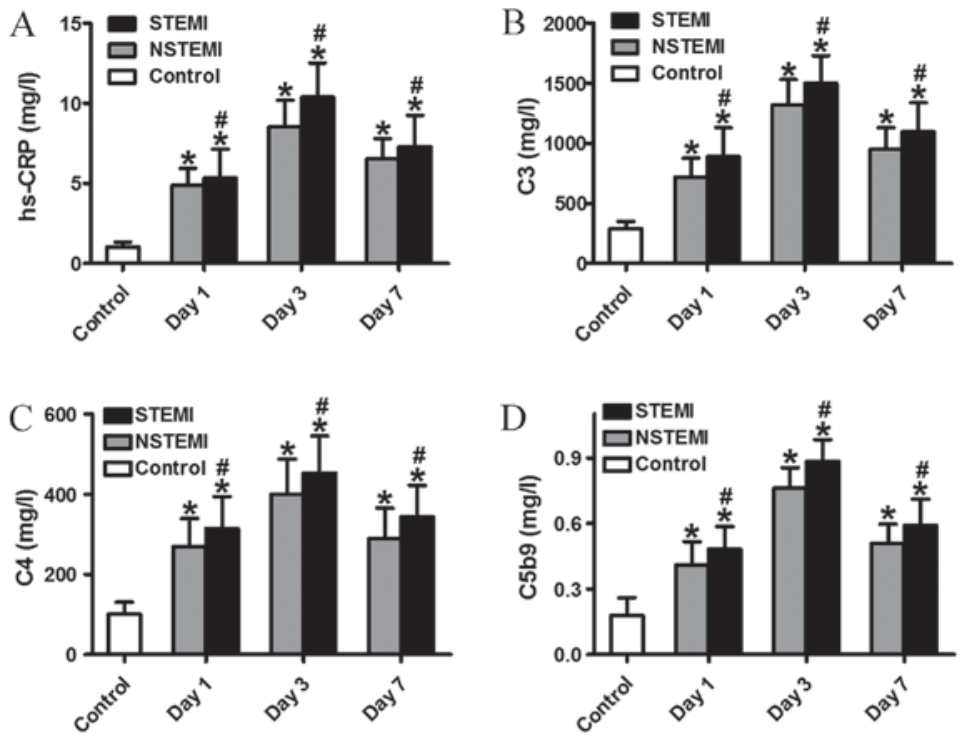

Figure 2. Comparison of serum levels of C3, C4, C5b9 and hs-CRP between the NSTEMI and STEMI subgroups and the control group. (A) Quantitative analysis data reveal that serum complement and hs-CRP levels of patients with STEMI and NSTEMI at day 3 were significantly higher than that of patients with NSTEMI and the control subjects. (B) Quantification of the serum C3 level. The serum C3 level of patients with STEMI and NSTEMI at day 3 was significantly higher than that of patients with NSTEMI and the control subjects. (C) Serum C4 levels of patients with STEMI and NSTEMI was measured at different time-points. The serum C4 level of patients with STEMI at day 3 was significantly higher than that of patients with NSTEMI and the control subjects. (D) ELISA detection of the C5b9 level of patients with STEMI and NSTEMI in the serum samples at days 1,3 and 7. Data are expressed as mean \pm standard deviation. "P $<0.05$ vs. control, ${ }^{,} \mathrm{P}<0.05$ vs. NSTEMI. AMI, acute myocardial infarction; STEMI, ST segment elevation MI; NSTEMI, non-ST segment elevation $\mathrm{MI}$; hs-CRP, high-sensitivity C-reactive protein.
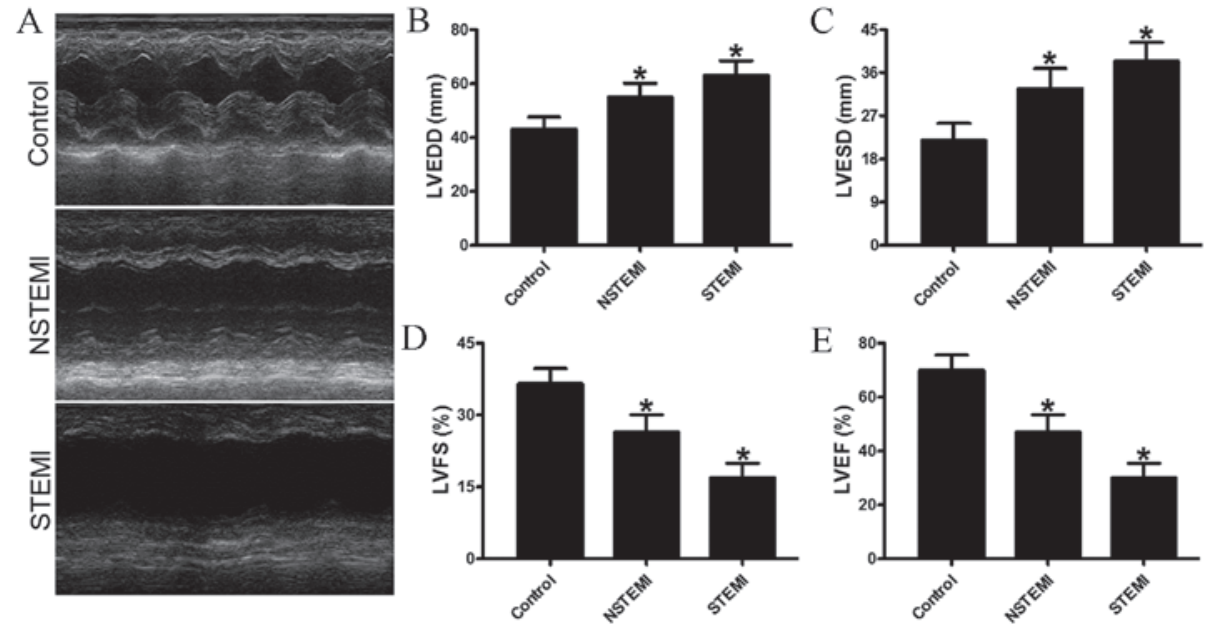

Figure 3. Echocardiographic evaluation of cardiac function. (A) Representative M-mode echocardiogram data of infarcted hearts of the control group, and NSTEMI and STEMI subgroups at day 7. (B and C) Comparison of LVEDD and LVESD between control group, NSTEMI subgroup and STEMI subgroup. (D and E) Quantitative analysis of LVFS and LVEF among the three groups. Compared with the control, the patients in the STEMI subgroup exhibited significantly accentuated heart function according to the LVEF values. ${ }^{~} \mathrm{P}<0.05$ vs. control. AMI, acute myocardial infarction; STEMI, ST segment elevation MI; NSTEMI, non-ST segment elevation MI; hs-CRP, high-sensitivity C-reactive protein; LVEDD, left ventricular end-diastolic diameter; LVESD, left ventricular end-systolic diameter; LVFS, left ventricular fractional shortening; LVEF, left ventricular ejection fraction.

LVFS and LVEF (Fig. 3D and E). Thus, the results indicate that cardiomyocyte injury was key in the cardiac remodeling and post infarction heart failure of patients in the STEMI group. Furthermore, these data reveal that activation of complement protein and hs-CRP are indicators of heart failure.

\section{Discussion}

In the current study, complement proteins and hs-CRP levels were demonstrated to be associated with the severity of myocardial injury in AMI patients. In addition, the high level of complement proteins and hs-CRP were demonstrated as a strong predictor of heart failure after AMI. The patients with STEMI exhibited significantly exacerbated ventricular dilation accompanied by progression to systolic dysfunction and heart failure (18).

Heart failure after AMI may be accompanied by a variety of effects, including increased apoptosis, decreased capillary density and increased hypertrophy. Notably, these effects occurred despite the complete activation of $\mathrm{C} 5 \mathrm{~b} 9$, which has 
a specific and important role in the response to AMI (19). A previous study revealed that injured or necrotic myocardial tissue releases subcellular constituents, such as mitochondria, which may be a trigger for complement activation pathways (20). In addition, increased complement proteins may impair regenerative processes to further aggravate myocardium injury (21). In uncomplicated myocardial infarction, the acute inflammatory reaction begins $4-8 \mathrm{~h}$ after onset, with the invasion of neutrophils from the perfused surrounding tissue, peaks within 3-4 days and returns to normal within 7 days (22). Therefore, peak complement and hs-CRP levels in patients with heart failure may be induced by ischemic necrosis and reperfusion injury may initiate this potent inflammatory stimulus (23). In addition, animal studies revealed that hs-CRP enhances ischemic tissue damage by a complement-dependent mechanism in the heart (24). Complement proteins have also been found to be deposited in atherosclerosis plaque (25).

In conclusion, the present study demonstrated that the serum levels of complement and hs-CRP increased rapidly in patients with AMI, particularly in early stage STEMI patients, and hs-CRP and activation of complement responses are immune inflammatory pathways that may lead to catastrophic consequences. However, there were certain limitations of the present study; it reflects a small sample of AMI patients, who were analyzed retrospectively and the long-term outcomes were not evaluated. Therefore, the present study may contribute to establishing biomarker-based approaches to rationally implement clinical therapeutic strategies for AMI.

\section{Acknowledgements}

The present study was supported by the Tianjin Municipal Bureau of Health for Science and Technology (grant no. 2014KZ052).

\section{References}

1. Du W, Tao H, Zhao S, He ZX and Li Z: Translational applications of molecular imaging in cardiovascular disease and stem cell therapy. Biochimie 116: 43-51, 2015.

2. Liu Y, Ye X, Mao L, Cheng Z, Yao X, Jia X, Mao D, Ou L, Li Z, Che Y, et al: Transplantation of parthenogenetic embryonic stem cells ameliorates cardiac dysfunction and remodelling after myocardial infarction. Cardiovasc Res 97: 208-218, 2013.

3. Frangogiannis NG: The inflammatory response in myocardial injury, repair, and remodelling. Nat Rev Cardiol 11: 255-265, 2014.

4. Liu N, Qi X, Han Z, Liang L, Kong D, Han Z, Zhao S, He ZX and $\mathrm{Li} Z$ : Bone marrow is a reservoir for cardiac resident stem cells. Sci Rep 6: 28739, 2016.

5. Cai M, Shen R, Song L, Lu M, Wang J,Zhao S, Tang Y, Meng X,Li Z and He ZX: Bone marrow mesenchymal stem Cells (BM-MSCs) improve heart function in swine myocardial infarction model through paracrine effects. Sci Rep 6: 28250, 2016.

6. Li Z, Lee A, Huang M, Chun H, Chung J, Chu P, Hoyt G, Yang P, Rosenberg J, Robbins RC, et al: Imaging survival and function of transplanted cardiac resident stem cells. J Am Coll Cardiol 53 1229-1240, 2009.

7. Janssen BJ, Huizinga EG, Raaijmakers HC, Roos A, Daha MR, Nilsson-Ekdahl K, Nilsson B and Gros P: Structures of complement component $\mathrm{C} 3$ provide insights into the function and evolution of immunity. Nature 437: 505-511, 2005.

8. Wysoczynski M, Solanki M, Borkowska S, van Hoose P, Brittian KR, Prabhu SD, Ratajczak MZ and Rokosh G: Complement component 3 is necessary to preserve myocardium and myocardial function in chronic myocardial infarction. Stem Cells 32: 2502-2515, 2014.
9. Zacho J, Tybjaerg-Hansen A, Jensen JS, Grande P, Sillesen H and Nordestgaard BG: Genetically elevated C-reactive protein and ischemic vascular disease. N Engl J Med 359: 1897-1908, 2008.

10. Melis JP, Strumane K, Ruuls SR, Beurskens FJ, Schuurman J and Parren PW: Complement in therapy and disease: Regulating the complement system with antibody-based therapeutics. Mol Immunol 67 (2 Pt A): 117-130, 2015.

11. Epelman S, Liu PP and Mann DL: Role of innate and adaptive immune mechanisms in cardiac injury and repair. Nat Rev Immunol 15: 117-129, 2015.

12. Thygesen K, Alpert JS, Jaffe AS, Simoons ML, Chaitman BR, White HD, Thygesen K, Alpert JS, White HD, Jaffe AS, et al; Joint ESC/ACCF/AHA/WHF Task Force for Universal Definition of Myocardial Infarction; Authors/Task Force Members Chairpersons; Biomarker Subcommittee; ECG Subcommittee; Imaging Subcommittee; Classification Subcommittee; Intervention Subcommittee; Trials \& Registries Subcommittee; Trials \& Registries Subcommittee; Trials \& Registries Subcommittee; Trials \& Registries Subcommittee; ESC Committee for Practice Guidelines (CPG); Document Reviewers: Third universal definition of myocardial infarction. J Am Coll Cardiol 60: 1581-1598, 2012.

13. White H, Thygesen K, Alpert JS and Jaffe A: Universal MI definition update for cardiovascular disease. Curr Cardiol Rep 16: 492, 2014.

14. Karahan Z, Uçaman B, Uluğ AV, Aydınalp Ö, Uğurlu M, Çevik K, Kaya İ and Öztürk Ö: Effect of hematologic parameters on microvascular reperfusion in patients with ST-segment elevation myocardial infarction treated with primary percutaneous coronary intervention. Angiology 67: 151-156, 2016.

15. Liu Z, Tang Q, Wen J, Tang Y, Huang D, Huang Y, Xie J, Luo Y, Liang M, Wu C, et al: Elevated serum complement factors 3 and 4 are strong inflammatory markers of the metabolic syndrome development: A longitudinal cohort study. Sci Rep 6: $18713,2016$.

16. Zethelius B, Berglund L, Sundström J, Ingelsson E, Basu S, Larsson A, Venge P and Arnlöv J: Use of multiple biomarkers to improve the prediction of death from cardiovascular causes. N Engl J Med 358: 2107-2116, 2008.

17. Dai S, Yuan F, Mu J, Li C, Chen N, Guo S, Kingery J, Prabhu SD, Bolli R and Rokosh G: Chronic AMD3100 antagonism of SDF-1alpha-CXCR4 exacerbates cardiac dysfunction and remodeling after myocardial infarction. J Mol Cell Cardiol 49: 587-597, 2010.

18. Frangogiannis NG: The immune system and the remodeling infarcted heart: Cell biological insights and therapeutic opportunities. J Cardiovasc Pharmacol 63: 185-195, 2014.

19. Thorp EB: Mechanisms of failed apoptotic cell clearance by phagocyte subsets in cardiovascular disease. Apoptosis 15: 1124-1136, 2010.

20. Giasuddin AS, ElMahdawi JM and ElHassadi FM: Serum complement $(\mathrm{C} 3, \mathrm{C} 4)$ levels in patients with acute myocardial infarction and angina pectoris. Bangladesh Med Res Counc Bull 33: 98-102, 2007

21. Mellbin LG, Bjerre M, Thiel S and Hansen TK: Complement activation and prognosis in patients with type 2 diabetes and myocardial infarction: A report from the DIGAMI 2 trial. Diabetes Care 35: 911-917, 2012.

22. Ribeiro DR, Ramos AM, Vieira PL, Menti E, Bordin OL Jr, Souza PA, Quadros AS and Portal VL: High-sensitivity C-reactive protein as a predictor of cardiovascular events after ST-elevation myocardial infarction. Arq Bras Cardiol 103: 69-75, 2014.

23. Timmers L, Pasterkamp G, de Hoog VC, Arslan F, Appelman Y and de Kleijn DP: The innate immune response in reperfused myocardium. Cardiovasc Res 94: 276-283, 2012.

24. Christenson E and Christenson RH: The role of cardiac biomarkers in the diagnosis and management of patients presenting with suspected acute coronary syndrome. Ann Lab Med 33: 309-318, 2013.

25. Wildgruber M, Swirski FK and Zernecke A: Molecular imaging of inflammation in atherosclerosis. Theranostics 3: 865-884, 2013. 